\title{
Characterisations of open multivalued linear operators
}

\author{
by \\ T. Álvarez (Oviedo)
}

\begin{abstract}
The class of all open linear relations is characterised in terms of the restrictions of the linear relations to finite-codimensional subspaces. As an application, we establish two results, the first of which shows that an upper semi-Fredholm linear relation retains its index under finite rank perturbations, and the second is a density theorem for lower bounded linear relations that have closed range. Results of Labuschagne and of Mbekhta about linear operators are covered.
\end{abstract}

1. Introduction. Several authors [10], [11], [13], [14] and [15] have proved characterisation results for open linear operators in order to establish density and perturbation theorems for various classes of linear operators of Fredholm theory. The aim of this paper is to find conditions under which results of this kind are true in the setting of multivalued linear operators.

In Section 2, the class of open linear relations is characterised by their action on finite-codimensional subspaces. As a consequence, we obtain two results, the first of which (Theorem 9) asserts that the index of an upper semiFredholm linear relation remains constant under finite rank perturbations, and the second (Theorem 11) shows that the class of lower bounded linear relations with closed range is dense in the class of upper semi-Fredholm linear relations with index not exceeding zero.

Notations. We follow the notation and terminology of the book [8]: $X, Y$ are normed spaces, $B_{X}$ the closed unit ball of $X$. Let $C(X)$ and $P(X)$ denote the finite-codimensional and the closed finite-codimensional subspaces of $X$ respectively.

A linear relation or multivalued linear operator $T: X \rightarrow Y$ is a mapping from a subspace $D(T) \subset X$, called the domain of $T$, into the collection of nonempty subsets of $Y$ such that $T\left(\alpha x_{1}+\beta x_{2}\right)=\alpha\left(T x_{1}\right)+\beta\left(T x_{2}\right)$ for all nonzero scalars $\alpha, \beta$ and $x_{1}, x_{2} \in D(T)$. The class of all such linear relations

2000 Mathematics Subject Classification: Primary 47A06.

Key words and phrases: multivalued linear operator, linear relation, open, semiFredholm, perturbation.

Supported in part by DGI (Spain), Proyecto BFM2001-1149. 
is denoted by $\operatorname{LR}(X, Y)$. If $T$ maps the points of its domain to singletons, then $T$ is said to be a linear operator.

The $\operatorname{graph} G(T)$ of $T \in \operatorname{LR}(X, Y)$ is $G(T):=\{(x, y) \in X \times Y: x \in D(T)$, $y \in T x\}$. Let $M$ be a subspace of $D(T)$. Then the restriction of $T$ to $M$, denoted by $\left.T\right|_{M}$, is defined by $G\left(\left.T\right|_{M}\right):=\{(m, y): m \in M, y \in T m\}$. For any subspace $M$ of $X$ such that $M \cap D(T) \neq \emptyset$, we write $\left.T\right|_{M \cap D(T)}=\left.T\right|_{M}$. The inverse of $T$ is the linear relation $T^{-1}$ defined by $G\left(T^{-1}\right):=\{(y, x) \in$ $Y \times X:(x, y) \in G(T)\}$. The subspace $N(T):=T^{-1}(0)$ is called the null space of $T$. If $N(T)$ is $\{0\}$ then $T$ is called injective, and $T$ is said to be surjective if its range $R(T):=T(D(T))$ equals $Y$.

The nullity and the deficiency of $T \in \operatorname{LR}(X, Y)$ are defined by $\alpha(T):=$ $\operatorname{dim} N(T)$ and $\beta(T):=\operatorname{dim} Y / R(T):=\operatorname{codim} R(T)$. If either $\alpha(T)$ or $\beta(T)$ is finite, then we define the index of $T$ by $i(T):=\alpha(T)-\beta(T)$.

For a given closed subspace $M$ of $X$ let $Q_{M}$ denote the quotient map from $X$ onto $X / M$. We shall denote $Q_{\overline{T(0)}}$ by $Q_{T}$. Clearly $Q_{T} T$ is a linear operator. For $x \in D(T),\|T x\|:=\left\|Q_{T} T x\right\|$ and the norm of $T$ is defined by $\|T\|:=\left\|Q_{T} T\right\|$.

A multivalued linear operator $T \in \operatorname{LR}(X, Y)$ is said to be closed if its graph is a closed subspace of $X \times Y$; continuous if for each neighbourhood $V$ in $R(T), T^{-1}(V)$ is a neighbourhood in $D(T)$, equivalently $\|T\|<\infty$; open if $T^{-1}$ is continuous, equivalently $\gamma(T)>0$ where $\gamma(T)$ is the minimum modulus of $T$ defined by

$$
\gamma(T):=\sup \{\lambda:\|T x\| \geq \lambda d(x, N(T)), x \in D(T)\} ;
$$

bounded below if it is injective and open; and bounded if it is continuous and $D(T)=X$.

Linear relations were introduced into functional analysis by J. von Neumann [16] motivated by the need of considering adjoints of nondensely defined linear differential operators which are considered by various authors (see, for example, [6] and [7]). Problems in optimisation and control also lead to the study of set valued maps and differential inclusions (see [4], [5], etc.) Studies of convex processes and tangent cones form part of the theory of convex analysis developed to deal with nonsmooth problems in viability and control theory, for example.

Other works on multivalued linear operators include the treatise on partial differential relations by Gromov [12], the application of multivalued methods to the solution of differential equations by Favini and Yagi [9] and several papers on linear relations of semi-Fredholm type and other related classes (see, for example, [1]-[3], [8] and [17]).

Throughout this paper, $X$ and $Y$ will denote infinite-dimensional normed spaces and $T$ will always denote an element of $\operatorname{LR}(X, Y)$ except where stated otherwise. 
2. Characterisations. We start this section by proving a result which gives equivalent conditions for a linear relation with finite-dimensional null space to be open.

Theorem 1. Let $\operatorname{dim} N(T)<\infty$. Then the following properties are equivalent:

(i) $T$ is open.

(ii) For every $M \in P(D(T)), R\left(\left.T\right|_{M}\right)$ is closed in $R(T)$ and $\left.T\right|_{M}$ is open if $M \cap N(T)=\{0\}$.

(iii) There exists $M \in P(D(T))$ such that $R\left(\left.T\right|_{M}\right)$ is closed in $R(T)$ and $\left.T\right|_{M}$ is injective and open.

(iv) There exists $M \in C(D(T))$ such that $R\left(\left.T\right|_{M}\right)$ is closed in $R(T)$ and $\left.T\right|_{M}$ is injective and open.

Proof. (i) $\Rightarrow$ (ii). Let $M$ be an arbitrary closed finite-codimensional subspace of $D(T)$. Then $M+N(T)$ is closed since $\alpha(T)<\infty$. To see that $R\left(\left.T\right|_{M}\right)$ is closed in $R(T)$, suppose $y_{k} \rightarrow y$ where $y \in T x$ and $y_{k} \in T m_{k}$ for $x \in D(T)$ and $\left(m_{k}\right) \subset M$; we shall prove that there is $m \in M$ for which $y \in T m$. Since $y \in T x \Leftrightarrow T x=y+T(0)$ and $y_{k} \in T m_{k} \Leftrightarrow T m_{k}=y_{k}+T(0)$ ([8, I.2.8]), we have $Q_{T} T m_{k}=Q_{T} y_{k} \rightarrow Q_{T} y=Q_{T} T x$ and noting that $0<\gamma(T)=\gamma\left(Q_{T} T\right)$ and $N(T)=N\left(Q_{T} T\right)\left(\left[8\right.\right.$, II.3.9]), it follows that $d\left(x-m_{k}, N(T)\right) \rightarrow 0$. Thus there exists $\left(n_{k}\right) \subset N(T)$ such that $m_{k}+n_{k} \rightarrow x \in M+N(T)$ (as $M+N(T)$ is closed). Hence $x=m+n$ for some $m \in M$ and $n \in N(T)$ and so we have $y+T(0)=T x=T(m+n)=T m+T n=T m+T(0)$ (as $n \in N(T) \Leftrightarrow T n=T(0)$ ), showing that $T m=y+T(0)$, equivalently $y \in T m$, as desired.

As the linear relations $T$ and $\left.T\right|_{M+N(T)}$ have the same null space, we derive from the definitions that $0<\gamma(T) \leq \gamma\left(\left.T\right|_{M+N(T)}\right)$.

Now assume that $N(T) \cap M=\{0\}$. Clearly $\left.T\right|_{M}$ is injective with $M \in$ $P(M+N(T))$ and thus by [8, II.3.18] there exists a continuous linear operator projection $P$ defined on $M+N(T)$ with range $M$ and null space $N(T)$. Hence for any $m \in M$,

$$
\begin{aligned}
\|m\| & =d\left(m, N\left(\left.T\right|_{M}\right)\right)=d(m, P(N(T))) \\
& =\inf \{\|P m-P n\|: n \in N(T)\} \leq\|P\| d(m, N(T)) .
\end{aligned}
$$

In consequence

$$
\begin{aligned}
0<\gamma(T) & \leq \gamma\left(\left.T\right|_{M+N(T)}\right) \\
& \leq \inf \left\{(\|P\|\|T m\|) / d\left(m, N\left(\left.T\right|_{M}\right)\right): m \in M \backslash N(T)\right\} \\
& =\|P\| \gamma\left(\left.T\right|_{M}\right)
\end{aligned}
$$

and thus $\left.T\right|_{M}$ is open.

The implications (ii) $\Rightarrow$ (iii) $\Rightarrow$ (iv) are obvious. 
(iv) $\Rightarrow\left(\right.$ i). Suppose $M \in C(D(T))$ and that $\left.T\right|_{M}$ is bounded below with closed range in $R(T)$. Since $\left.T\right|_{M}$ is injective, we can choose as $F$ a complement of $M \oplus N(T)$ in $D(T)$. Thus

(1) $\left.T\right|_{M+F}$ is injective.

Now, since $\operatorname{dim} R(T) / R\left(\left.T\right|_{M}\right) \leq \operatorname{dim} D(T) / M$ by [8, I.6.1] and $M \in$ $C(D(T))$ we derive that

$$
R\left(\left.T\right|_{M}\right) \text { is a closed finite-codimensional subspace of } D\left(\left(\left.T\right|_{M+F}\right)^{-1}\right)
$$

and as $\left(\left.T\right|_{M}\right)^{-1}$ is continuous and clearly $\left(\left.T\right|_{M}\right)^{-1}=\left.\left(\left.T\right|_{M+F}\right)^{-1}\right|_{T M}$ we conclude from (2) and [8, II.3.19] that $\left(\left.T\right|_{M+F}\right)^{-1}$ is continuous, that is,

(3) $\left.T\right|_{M+F}$ is open.

Finally, we conclude that $T$ is open since

$$
\begin{aligned}
0 & <\gamma\left(\left.T\right|_{M+F}\right)=\inf \{\|T x\| /\|x\|: x \in M+F\} \\
& \leq \inf \{\|T x\| / d(x, N(T)): x \in M+F\} \\
& =\inf \{\|T x\| / d(x, N(T)): x \in D(T) \backslash N(T)\}=\gamma(T) .
\end{aligned}
$$

(Here we have combined (1) and (3)).

This theorem generalises the corresponding result for linear operators of Labuschagne $[13,7]$.

Our next objective is to extend the above theorem to arbitrary linear relations. To this end, we shall use some auxiliary results concerning canonical factorisation and graph operators which we now present.

Definition 2 ([8, V.13.1]). The injective component $\widehat{T}$ of $T$ is the linear relation $\widehat{T} \in \operatorname{LR}(X / N(T), Y)$ given by $G(\widehat{T}):=\{([x], y):(x, y) \in G(T)\}$. The representation $T=\widehat{T} Q_{N(T)}$ is referred to as the canonical factorisation of $T$.

It is trivial that $T x=\widehat{T}[x](x \in D(T))$ and that $X / N(T)$ is a normed space if and only if $N(T)$ is closed, for example if $T$ is closed.

Proposition 3. If $N(T)$ is closed, then $T$ is open if and only if so is $\widehat{T}$.

Proof. Since $\widehat{T}^{-1}=Q_{N(T)} T^{-1}=Q_{T^{-1}(0)} T^{-1}$, we obtain $\left\|\widehat{T}^{-1}\right\|=$ $\left\|Q_{N(T)} T^{-1}\right\|=\left\|T^{-1}\right\|$ and as $\left\|T^{-1}\right\|=\gamma(T)$ by [8, II.2.5], the desired result holds.

Definition 4 ([8, IV.3.1]). Given $T \in \operatorname{LR}(X, Y)$, let $X_{T}$ denote the vector space $D(T)$ normed by $\|x\|_{T}:=\|x\|+\|T x\|, x \in D(T)$. The graph operator $G_{T}$ is defined by $D\left(G_{T}\right):=X_{T}$ and $G_{T} x:=x, x \in X_{T}$.

Proposition 5 ([8, IV.3.2 and IV.3.10]). $T G_{T}$ is a continuous everywhere defined linear relation such that $N\left(T G_{T}\right)$ is a closed subspace of $X_{T}$ and $\gamma\left(T G_{T}\right)=\gamma(T) /(1+\gamma(T))($ where $\infty / \infty:=1)$. 
THEOREM 6. $T$ is open if and only if there exists $M \in C(D(T))$ such that $R\left(\left.T\right|_{M}\right)$ is closed in $R(T)$ and $\left.T\right|_{M}$ is open.

Proof. Let $M$ be a finite-codimensional subspace of $D(T)$ such that $R\left(\left.T\right|_{M}\right)$ is closed in $R(T)$ and $\gamma\left(\left.T\right|_{M}\right)>0$. Then $M+N(T) \in C(D(T))$, $T M=T(M+N(T))$ and $N\left(\left.T\right|_{M}\right) \subset N(T)=N\left(\left.T\right|_{M+N(T)}\right)$ and thus it follows immediately from the definitions that

$$
\gamma\left(\left.T\right|_{M}\right) \leq \gamma\left(\left.T\right|_{M+N(T)}\right) .
$$

Let us consider two cases for $N(T)$ :

Case 1: $N(T)$ closed. First we note that as $N(T) \subset M+N(T)$, the injective component of $\left.T\right|_{M+N(T)}$ coincides with $\left.\widehat{T}\right|_{(M+N(T)) / N(T)}$. Furthermore, $(M+N(T)) / N(T)$ is a finite-codimensional subspace of $D(\widehat{T})$ and

$$
\widehat{T}((M+N(T)) / N(T))=T(M+N(T))=T M,
$$

which is closed in $R(T)=R(\widehat{T})$ and so $\gamma\left(\left.\widehat{T}\right|_{(M+N(T)) / N(T)}\right)=\gamma\left(\left.T\right|_{M+N(T)}\right)$ $>0$ by Proposition 3 and $(*)$. Now, Theorem 1 shows that $\gamma(\widehat{T})>0$ and again by Proposition 3 we conclude that $T$ is open.

Case 2: $N(T)$ not closed. We consider the linear relation $T G_{T}$. By Proposition 5, $T G_{T}$ has closed null space and it is easy to see that

$$
G_{T}^{-1}(M+N(T)) \in C\left(X_{T}\right) \text { and } T G_{T}\left(G_{T}^{-1}(M+N(T))\right)=T M,
$$

which is closed in $R(T)=R\left(T G_{T}\right)$. Now, if we can show that the restriction $\left.T G_{T}\right|_{G_{T}^{-1}(M+N(T))}$ is open, it will follow from Case 1 that $\gamma\left(T G_{T}\right)>0$ and then applying Proposition 5 we will conclude that $T$ is open.

To prove the openness of $\left.T G_{T}\right|_{G_{T}^{-1}(M+N(T))}$ it is enough to observe that

$$
\gamma\left(\left(\left.T\right|_{M+N(T)}\right)\left(\left.G_{T}\right|_{G_{T}^{-1}(M+N(T))}\right)\right) \leq \gamma\left(\left.T G_{T}\right|_{G_{T}^{-1}(M+N(T))}\right)
$$

by [8, II.3.11] and also that $\left.T\right|_{M+N(T)}$ is open by (*).

The converse is clear.

In the particular case of linear operators the previous theorem was obtained by Labuschagne $[13,8]$.

Definition 7. A linear relation $T$ is called upper semi-Fredholm if $\gamma(T)>0, \alpha(T)<\infty$ and $R(T)$ is closed. The corresponding class of linear relations will be abbreviated $\operatorname{USF}(X, Y)$.

We shall use the above characterisation results to investigate the stability of upper semi-Fredholm linear relations under finite rank perturbations. We also recall the following finite-dimensional extension lemma for linear relations. 
Lemma 8 ([8, V.15.5]). Let $S, T \in \operatorname{LR}(X, Y)$ and let $S$ be an extension of $T$ (that is, $\left.S\right|_{D(T)}=T$ ) such that $\operatorname{dim} D(S) / D(T):=n<\infty$. If $T$ has an index, then $i(S)=i(T)+n$.

Theorem 9. Let $T \in \operatorname{USF}(X, Y)$ and let $S$ be a continuous finite rank linear relation such that $D(T) \subset D(S)$ and $S(0) \subset \overline{T(0)}$. Then $T+S \in$ $\operatorname{USF}(X, Y)$ and $i(T+S)=i(T)$ if $i(T)$ exists.

Proof. We assume without loss of generality that $D(T)=X$. Let $M:=$ $N(S)$. Since $S(0)$ is closed (as $R(S)$ is finite-dimensional), $N(S)=N\left(Q_{S} S\right)$ is a closed finite-codimensional subspace of $X$. Observing that as $T$ has closed range with $S(0) \subset \overline{T(0)}$ by hypothesis and $T M$ is closed, it follows from Theorem 1 that $T M=(T+S) M$ is a closed subspace of $Y$. Furthermore, since $m \in N\left(\left.(T+S)\right|_{M}\right)$ if and only if $(T+S) m=(T+S)(0)$ for $m \in M$ if and only if $m \in N\left(\left.T\right|_{M}\right)$, we have $N\left(\left.T\right|_{M}\right)=N\left(\left.(T+S)\right|_{M}\right)$ and so it follows immediately from the definitions that $\gamma\left(\left.T\right|_{M}\right)=\gamma\left(\left.(T+S)\right|_{M}\right)$. Hence, if we can show that $\left.T\right|_{M}$ is open, it will follow from Theorem 6 that $T+S$ is also open.

To see that $\gamma\left(\left.T\right|_{M}\right)>0$, we note that since the linear relations $T$ and $\left.T\right|_{M+N(T)}$ have the same null space, $\gamma(T) \leq \gamma\left(\left.T\right|_{M+N(T)}\right)$. Moreover, as $M \in P(M+N(T))$ there exists a continuous linear projection $P$ from $M+N(T)$ onto $M$ and for any $m \in M$ we have

$$
d\left(m, N\left(\left.T\right|_{M}\right)=\inf \{\|P m-P n\|: n \in N(T)\} \leq\|P\| d(m, N(T)) .\right.
$$

Consequently, $0<\gamma(T) \leq \gamma\left(\left.T\right|_{M+N(T)}\right) \leq\|P\| \gamma\left(\left.T\right|_{M}\right)$ and hence $\left.T\right|_{M}$ is open, as desired.

Since $(T+S) M$ is closed and $\operatorname{dim} R(T+S) /(T+S) M \leq \operatorname{dim} X / M$ $<\infty$ we see that $R(T+S)$ is a closed subspace of $Y$, while the property $\alpha(T+S)<\infty$ is trivially obtained by [8, V.2.4, V.3.2 and V.5.1]. Finally, assume that $i(T)$ exists and let $\operatorname{dim} X / M:=n<\infty$. Combining Lemma 8 with $R\left(\left.T\right|_{M}\right)=R\left(\left.(T+S)\right|_{M}\right)$ and the closedness of both $R(T)$ and $R(T+S)$ yields $i(T)=i\left(\left.T\right|_{M}\right)+n=i\left(\left.(T+S)\right|_{M}\right)+n=i(T+S)$. Hence the result follows.

As a consequence of Theorem 9 we give a density result for lower bounded linear relations that have closed range. We also need the following elementary lemma.

Lemma 10. Let $M$ be a closed subspace of $X$, and let $N \subset X$ be a subspace such that $M \subset N$. Then $N$ is closed if and only if $N / M$ is closed.

Theorem 11. Let $T \in \operatorname{USF}(X, Y)$ with $i(T) \leq 0$. Then for every $\varepsilon>0$ there exists $T_{\varepsilon} \in \operatorname{LR}(X, Y)$ such that $T_{\varepsilon}$ is bounded below, $R\left(T_{\varepsilon}\right)$ is closed and $\left\|T_{\varepsilon}-T\right\| \leq \varepsilon$. 
Proof. Since $T$ has closed range, it follows from Lemma 10 that $R\left(Q_{T} T\right)$ $=R(T) / \overline{T(0)}$ is also closed and $\beta(T)=\beta\left(Q_{T} T\right)$. Furthermore, as $\gamma(T)>0$ and $N(T)$ is closed, applying [8, II.3.9], we obtain $\gamma(T)=\gamma\left(Q_{T} T\right)$ and $N(T)=N\left(Q_{T} T\right)$. Consequently, $Q_{T} T$ is an upper semi-Fredholm linear relation such that $N(T)=N\left(Q_{T} T\right)$ and $i(T)=i\left(Q_{T} T\right) \leq 0$. Choose a basis $\left\{x_{1}, \ldots, x_{n}\right\}$ for $N(T)$ with $\left\|x_{i}\right\|=1(1 \leq i \leq n)$ and let $\left[y_{1}\right], \ldots,\left[y_{n}\right]$ be linearly independent elements of $Y / \overline{T(0)}$ whose linear span intersects $R\left(Q_{T} T\right)$ only in $\{[0]\}$ and $\left\|\left[y_{i}\right]\right\|=1(1 \leq i \leq n)$. As $Q_{T}$ is surjective and $Q_{T} B_{Y}=B_{Q_{T} Y}$ we can select $y_{1}, \ldots, y_{n}$ linearly independent in $B_{Y}$ such that $Q_{T} y_{i}=\left[y_{i}\right](1 \leq i \leq n)$. By the Hahn-Banach theorem there exists a set $\left\{x_{1}^{\prime}, \ldots, x_{n}^{\prime}\right\}$ of linear functionals in $X^{\prime}$ satisfying $\left\|x_{i}^{\prime}\right\|=1$ and $x_{i}^{\prime}\left(x_{i}\right)=\delta_{i j}(1 \leq i, j \leq n)$.

Let $\varepsilon>0$. Define

$$
F_{\varepsilon} x:=(\varepsilon / n) \sum_{i=1}^{n} x_{i}^{\prime}(x) y_{i} \quad(x \in X) .
$$

Then it is clear that $F_{\varepsilon}$ is a bounded finite rank linear operator whose norm does not exceed $\varepsilon$. We shall verify that $T_{\varepsilon}:=T+F_{\varepsilon}$ is a lower bounded linear relation with closed range and $\left\|T_{\varepsilon}-T\right\| \leq \varepsilon$.

By Theorem 9, $T_{\varepsilon} \in \operatorname{USF}(X, Y)$. Now, as $T(0)=T_{\varepsilon}(0)=\left(T_{\varepsilon}-T\right)(0)$, it follows that $Q_{T}=Q_{T+F_{\varepsilon}}=Q_{T_{\varepsilon}-T}$ and hence $Q_{T_{\varepsilon}+F_{\varepsilon}}\left(T+F_{\varepsilon}\right)=Q_{T} T+$ $Q_{T} F_{\varepsilon}$. From this we obtain $\left\|T_{\varepsilon}-T\right\|:=\left\|Q_{T_{\varepsilon}-T}\left(T_{\varepsilon}-T\right)\right\|=\left\|Q_{T} F_{\varepsilon}\right\| \leq \varepsilon$ and to prove the injectivity of $T_{\varepsilon}$ it is enough to show that $Q_{T} T+Q_{T} F_{\varepsilon}$ is an injective linear operator (as trivially $N\left(T+F_{\varepsilon}\right) \subset N\left(Q_{T+F_{\varepsilon}}\left(T+F_{\varepsilon}\right)\right)$ ). But $\left(Q_{T} T+Q_{T} F_{\varepsilon}\right) x=0 \Rightarrow Q_{T} F_{\varepsilon} x=-Q_{T} T x \in R\left(Q_{T} T\right) \cap \operatorname{span}\left\{\left[y_{1}\right], \ldots,\left[y_{n}\right]\right\}$ $=\{[0]\} \Rightarrow x \in N\left(Q_{T} T\right) \cap N\left(Q_{T} F_{\varepsilon}\right) \Rightarrow x=0$, that is, $Q_{T} T+Q_{T} F_{\varepsilon}$ is injective, as required.

Corollary 12. Let $T \in \operatorname{LR}(X, Y)$ be closed, where $X$ and $Y$ are $B a$ nach spaces, and suppose that $R(T)$ is closed, $\operatorname{dim} N(T)<\infty$ and $i(T) \leq 0$. Then for every $\varepsilon>0$ there exists $T_{\varepsilon} \in \operatorname{LR}(X, Y)$ that is closed, injective with closed range and such that $\left\|T_{\varepsilon}-T\right\| \leq \varepsilon$.

Proof. The closed graph and open mapping theorems for multivalued linear operators [8, III.5.3 and III.5.4] assert that for closed linear relations $S$ between Banach spaces, $S$ is open if and only if $R(S)$ is closed. Combining this with Theorem 11 shows that the linear relation $T_{\varepsilon}$ obtained as in Theorem 11 is injective, has closed range and $\left\|T_{\varepsilon}-T\right\| \leq \varepsilon$. Furthermore, as $T$ is closed and $F_{\varepsilon}$ is a bounded linear operator, $T_{\varepsilon}=T+F_{\varepsilon}$ is closed by [8, II.5.16].

For the particular case of linear operators, the above corollary was proved (independently) by Galaz-Fontes $[10,2.1]$ for the case where $X=Y$ and $T$ 
is bounded and by Mbekhta [14,3] for the case when $T$ is closed. Our proof is different.

Acknowledgements. We thank the referee for several valuable comments.

\section{References}

[1] T. Álvarez, R. W. Cross and D. Wilcox, Multivalued Fredholm type operators with abstract generalised inverses, J. Math. Anal. Appl. 261 (2001), 403-417.

[2] - - - - , Quantities related to upper and lower semi-Fredholm type linear relations, Bull. Austral. Math. Soc. 66 (2002), 275-289.

[3] -, -, -, Adjoint characterisations of quasi-weakly compact linear relations, J. Math. Anal. Appl. 277 (2003), 257-271.

[4] J. P. Aubin and A. Cellina, Differential Inclusions, Springer, New York, 1984.

[5] F. H. Clarke, Optimization and Nonsmooth Analysis, Wiley, Toronto, 1983.

[6] E. A. Coddington, Multivalued Operators and Boundary Value Problems, Lecture Notes in Math. 183, Springer, Berlin, 1971.

[7] E. A. Coddington and A. Dijksma, Selfadjoint subspaces and eigenfunction expansions for ordinary differential subspaces, J. Differential Equations 20 (1976), 473526.

[8] R. W. Cross, Multivalued Linear Operators, Monographs Textbooks Pure Appl. Math. 213, Dekker, New York, 1998.

[9] A. Favini and A. Yagi, Multivalued linear operators and degenerate evolution equations, Ann. Mat. Pura Appl. (4) 163 (1993), 353-384.

[10] F. Galaz-Fontes, Approximation by semi-Fredholm operators, Proc. Amer. Math. Soc. 120 (1994), 1219-1222.

[11] S. Goldberg, Unbounded Linear Operators, McGraw-Hill, New York, 1966.

[12] M. Gromov, Partial Differential Relations, Springer, Berlin, 1986.

[13] L. E. Labuschagne, The perturbation of relatively open operators with reduced index, Math. Proc. Cambridge Philos. Soc. 112 (1992), 385-402.

[14] M. Mbekhta, Remarques sur la structure interne des composantes connexes semiFredholm, Studia Math. 119 (1994), 251-256.

[15] - On the stability of semi-Fredholm operators, J. Operator Theory 35 (1996), 191-201.

[16] J. von Neumann, Functional Operators: The Geometry of Orthogonal Spaces, Vol. 2, Ann. of Math. Stud. 22, Princeton Univ. Press, Princeton NJ, 1950.

[17] D. Wilcox, Multivalued Semi-Fredholm Operators in Normed Linear Spaces, Ph.D. thesis, Univ. of Cape Town, 2001.

Department of Mathematics

University of Oviedo

33007, Oviedo, Spain

E-mail: seco@uniovi.es

Received June 23, 2004

Revised version January 25, 2005 